\title{
HIGH EFFICIENCY COMPACT SOURCE OF MONOCHROMATIC TUNABLE X-RAY RADIATION ON BASE OF ELECTRON ACCELERATOR WITH MODERATE PARTICLE ENERGY
}

\author{
V.K.Grishin ${ }^{+}$, S.P.Likhachev, Moscow Lomonosov State University, Moscow, Russia \\ N.N.Nasonov, Belgorod State University, Belgorod, Russia
}

\begin{abstract}
Project of high efficiency compact monochromatic tunable X-ray radiation source is considered. Special magnet scheme for parametric $\mathrm{x}$-ray radiation (PXR) generation arising in vicinity of Brag direction while an electron beam multiple hits a crystal target is proposed. Peculiarities of magnet system permitting stable electron circling are discussed. First results of computer simulation are reported.
\end{abstract}

\section{INTRODUCTION}

In present several kinds of relativistic electron coherent emission processes in condensed media are been studied as a candidate for quasimonochromatic highly directed $\mathrm{X}$-ray source creation. One of the most effective possible $\mathrm{x}$-ray sources may be basing on the parametric $\mathrm{x}$-ray radiation (PXR) of relativistic electrons crossing a crystal [1-3]. The essential shortcoming of this emission mechanism connected with a small PXR intensity is stipulating the attempts to test the different schemes, which would permit to increase the photon yield.

How is it possible to raise PXR intensity of electrons? Evident step consists in increase of target thickness. But this way is not efficient. At first that produces increasing the angular dispersion of electrons. A permissible angular divergence of particles can not exceed here the magnitude of cone angle of radiation flow that is about $1 / \gamma$ where $\gamma$ is Lorenz factor of incident electrons. Besides the self-absorption of generated photons becomes very considerable in the substance of thick target.

Therefore this work is devoted to study of the possibility to create an effective $\mathrm{x}$-ray source based on PXR by low relativistic electrons crossing repeatedly a thin crystalline target. The proposed arrangement permits generate the monochromatic, polarized, and tunable X-ray radiation, which can be very widely used in the different scientific and other applications.

\section{PRACTICAL SCHEME. MAGNETIC SYSTEM}

Thus, a special scheme of PXR generation in which

+ Email: grishin@depni.npi.msu.su electrons are multiple crossing a crystalline target, is been considered. Low relativistic electrons are been used what allows obtain a compact size of layout.

The suggested experimental setup is presented in Fig.1.

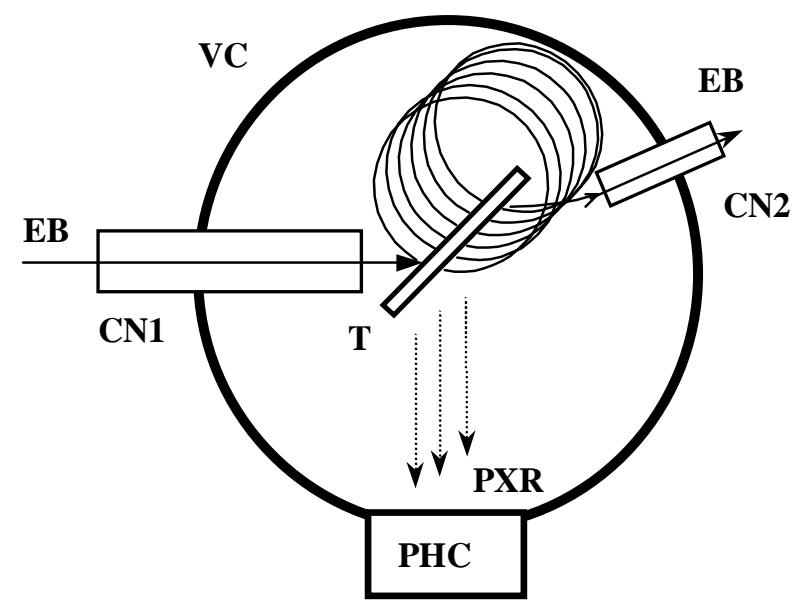

Figure 1.The scheme of experimental setup. VC is a vacuum chamber, $\mathrm{T}$ is a crystalline target, $\mathrm{EB}$ is an electron beam, PHC is photon channel for PXR, CH1 and $\mathrm{CH} 2$ are channels for injection and removal of electrons.

Here in a vacuum chamber $\mathrm{VC}$, a crystalline target $\mathrm{T}$ is installed. The target is immersed in a magnetic field. The electron beam EB is injecting through a special channel $\mathrm{CH} 1$ in the work volume. Electrons circling in the magnet field are hitting the target several times. Then they are been removed through a channel $\mathrm{CH} 2$. PXR generated by electrons is been taken out through a photon channel PCH.

A gist of proposal is to force electrons to do stable multiple crossing of target after preceded ones. For this the special configuration of magnetic field is considered. Here a magnetic field must carry out two functions. The first one is ensuring a stable circulation of electrons with its properly focused fall on the target. The second one is a necessity to shift the rotating particles along the target. A suitable field configuration can be creating by means of magnetic poles of a simple form. These are two parallelepipeds placed over and below the target and stretched along it. The poles are slightly shifted across target plane. In this case the fields dispersed on poles 
ridges are ensuring spatial focusing of circling particles. Due to a displacement of poles, mean magnitudes of fields are not equal left and right the target, and the latter provides a lateral drift of particles. By results of our previous works devoted researches of efficiency increase of usual bremsstrahlung source, the similar magnetic configurations provides reliable focusing of particle [4].

\section{COMPUTER SIMULATION. EMISSION YIELD CALCULATION.}

For testing the considered scheme we are applying a method of computer modeling. The whole procedure of modeling by a natural way breaks up to two parts. Change of energy of particles and their dispersion are defined basically by ionization losses of particles in substance of a target. Casual drawing of these events and accompanying radiation of bremsstrahlung quanta, and also dynamics of trajectories of particles in a magnetic field are been simulated on the basis of GEANT program library [5]. The process PXR is been simulated with the help of the additional software package, in which the casual drawing of the acts of radiation PXR photons is been described proceeding from the following physical representations.

PXR is a process of a fast particle coulomb field coherent scattering by a system of crystalline atomic planes. This emission has been studied well theoretically. Therefore we are calculating the spectrum of the photons emitted by an electron beam in a thin crystal using the well-known formula for PXR spectral-angular distribution (see for example [6]). In our calculations we are neglecting the dynamical diffraction effects as well as photoabsorption because of small enough emitting electron energy and crystal thickness, but taking into account the influence of emitting particle multiple scattering in a target and a finite angular size of a radiation collimator. The gaussian distribution function over beam electron scattering angles is been used. The final beam angular spread for the previous interaction of an electron beam with a target is been applied as an initial one for the subsequent interaction because the internal magnetic field in our scheme does not increase a beam angular spread. The orientation angle (see the emission geometry presented in Fig.1) is been selected to be equal to a half of inverse Lorentz factor that corresponds to the maximum PXR yield to small collimator. Notice, that the character PXR here poorly depends on energy of electrons, and is defined by length of radiation wave and Bragg angle. Therefore intensity of PXR of each particle grows proportionally to number of its target crossings within the limits of indicated angle.

Specify the basic physical parameters of modeling. The project is oriented on using of MSU $2.4 \mathrm{MeV}$ electron $\mathrm{CW}$ linear accelerator which produces the continuous beam current up to $1 \mathrm{~mA}$ and beam radius of $\approx 1 \mathrm{~mm}$ (CW devices are the most suitable kinds of accelerators because they can produce a very high mean intensity of $\mathrm{PXR}$ ). Hence Lorenz factor is $\approx 6$, and angle of radiation cone is about $10^{\circ}$.

In an initial configuration Bragg angle is chosen as $45^{\circ}$. A silicon crystal is chosen as a material of target. The crystalline target plane is made in such a manner that the reciprocal lattice vector $\langle 111\rangle$ is lying along its surface. Basing on the facts mentioned above, the estimates indicate that the optimal value of silicon target thickness can be no more than 20-30 mkm.

The maximal magnitude of a mean current of electron beam can be estimated from following reasons. According to work [7], average maximal beam current is of order $0.5 \mathrm{~A}$ at beam radius of $1 \mathrm{~mm}$. Therefore, taking into account displacement of particles along a target at their rotations, it is possible to admit that the planned for using accelerator can be under operation in limiting mode.

In Fig. 2 and 3 the first results of simulation of particle dynamics are presented.

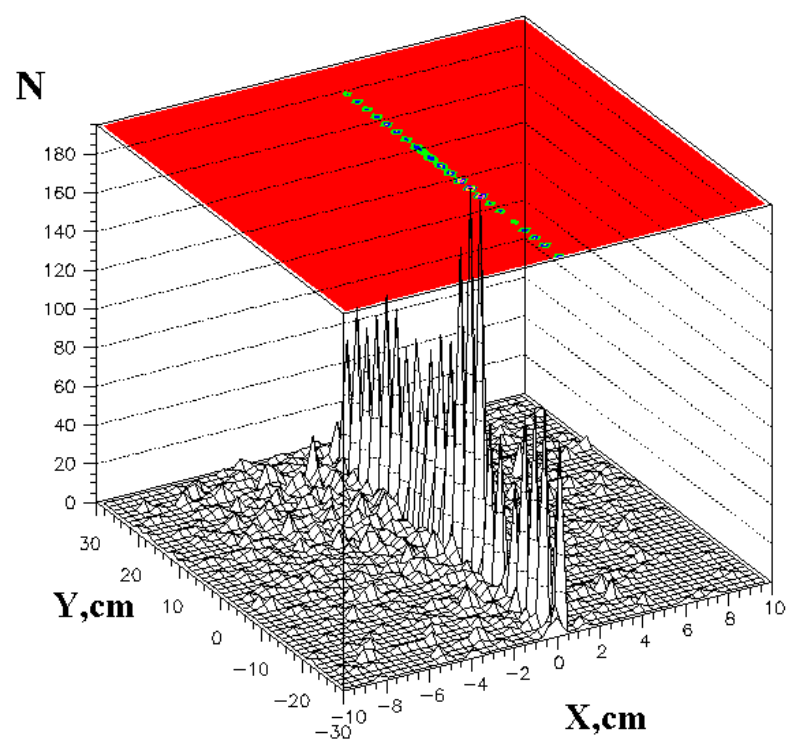

Figure 2. Spatial distribution of intersection points of circling electron trajectories with a target plane (demonstration test with the increased step of cross drift of electrons). $\mathrm{X}$-axis is vertically directed, $\mathrm{Y}$-axis is stretched along a target.

By this data, the particles can make some tens circulation's, and not less than one- two tens of revolutions are made within the limits of a demanded angle of radiation. With that the mean PXR intensity reaches up ten $\mathrm{W} / \mathrm{Sr}$. 


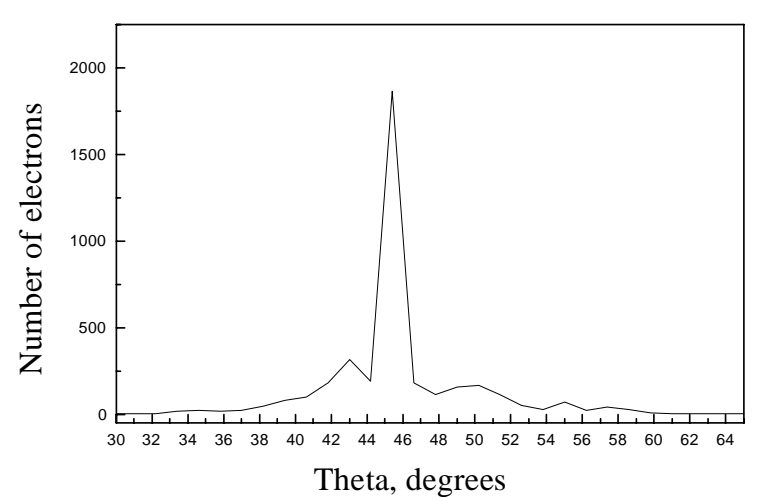

Figure 3. Angular (near to Bragg angle) distribution of electrons crossing a target plane.

\section{CONCLUSION}

Thus the developed analysis shows the possibility to create an effective compact $x$-ray source consisting of a linear electron accelerator with particle energy of the order of about units $\mathrm{MeV}$, thin crystalline target providing the generation of quasimonochromatic highly directed tunable x-rays on the base of PXR mechanism and the magnetic system providing the multiple interaction of emitting particles with a target.
Moderate consumed power and compact sizes of similar devices (compare, for example, with [7]) open reliable prospects of their wide and various applications.

\section{REFERENCES}

[1] M. Ter-Mikaelian. High energy electromagnetic processes in condensed media (Wiley-Interscience. NewYork, 1972 )

[2] V.Baryshevsky, I.Feranchuk. Zh.Eksp.Teor.Fiz. 61 (1971) 944

[3] G.Garibian, Y.Shi. Zh.Eksp.Teor.Fiz. 61 (1971) 930 [4] V.Grishin, B.Ishkhanov, S.Likhachev et al., Proc.of Int. Conf. PAC97, APS-IEEE, 1997. P.3866.

[5] R.Brun, F. Bruyant, M. Maine, GEANT3 (User's Guide), DATA HENDLING, CERN Geneva, Switzerland.

[6] N.Nasonov, A.Safronov. Proc.Int.Symp. RREPS-93, Oct. 1993, Tomsk, Russia.

[7] A.Andriyashkin, V. Kaplin, M.Pristrup et al., Appl. Phys. Lett., v. 72, N 11, p.1385 (1988). 